\title{
Suspected Necrotizing Enterocolitis
}

National Cancer Institute

\section{Source}

National Cancer Institute. Suspected Necrotizing Enterocolitis. NCI Thesaurus. Code C101273.

The diagnosis assigned when a health care practitioner feels strongly the patient has necrotizing enterocolitis, but lacks definitive proof. 